\title{
SURFACES IN MÖBIUS GEOMETRY
}

\author{
CHANGPING WANG* \\ To Chiu and Ya-Ya
}

\section{§ 0. Introduction}

Our purpose in this paper is to give a basic theory of Möbius differential geometry. In such geometry we study the properties of hypersurfaces in unit sphere $S^{n}$ which are invariant under the Möbius transformation group on $S^{n}$.

Since any Möbius transformation takes oriented spheres in $S^{n}$ to oriented spheres, we can regard the Möbius transformation group $G_{n}$ as a subgroup $M G_{n}$ of the Lie transformation group on the unit tangent bundle $U S^{n}$ of $S^{n}$. Furthermore, we can represent the immersed hypersurfaces in $S^{n}$ by a class of Lie geometry hypersurfaces (cf. [9]) called Möbius hypersurfaces. Thus we can use the concepts and the techniques in Lie sphere geometry developed by U. Pinkall ([8], [9]), T. Cecil and S.S. Chern [2] to study the Möbius differential geometry.

We will study in detail the surface theory in Möbius geometry. The same method can be easily generalized to high dimensional cases. We give a complete Möbius invariant system for any immersed surface without umbilic point in $S^{3}$ which determines this surface up to Möbius transformations. Moreover, given any such Möbius invariant system we can obtain the corresponding Möbius surface by solving a linear PDE determined by this invariant system.

An immediate application of our theory is the classification of Dupin surfaces in $E^{3}$ under the conformal transformation group. We show that up to the conformal transformations a Dupin surface in $E^{3}$ is a part of a revolution torus, a right circular cylinder or a right circular cone.

Möbius geometry has a close relation with the famous Willmore conjecture. An elegant application of Möbius geometry was given by R. L.

Received March 27, 1991.

*Supported by Technische Universität Berlin. 
Bryant [1] in order to study the Willmore surfaces in $S^{3}$. In this paper we give an expression of the Euler-Lagrange equation for Willmore surfaces in terms of Möbius invariants.

This paper is organized as follows:

$\S 0$. Introduction

$\S 1$. Lie sphere geometry

$\S 2$. Möbius surfaces and Möbius transformations

$\S 3$. Möbius invariants for Möbius surfaces

$\S 4$. Fundamental theorems for Möbius surfaces

$\S 5$. Classification of Dupin surfaces in $E^{3}$ under the conformal transformation group

I would like to thank Professor S. S. Chern for his direction and Professor U. Pinkall for helpful discussion.

\section{§1. Lie sphere geometry}

In this section we review some basic concepts and facts concerning the surfaces in Lie sphere geometry. For detail we refer to Cecil and Chern [2] and Pinkall [9].

\subsection{Oriented spheres and Lie transformations}

Let $S^{3}$ be the unit sphere in $E^{4}$ and $U S^{3}$ the unit tangent bundle of $S^{3}$. An oriented sphere in $U S^{3}$ is a mapping $(x, n): S^{2} \rightarrow U S^{3}$ such that $x: S^{2} \rightarrow S^{3}$ is an umbilic sphere and $n$ is one of the unit normal vector fields along $S^{2}$. When $x: S^{2} \rightarrow S^{3}$ shrinks to a point $x$, we define $n$ to be the inclusion $i_{x}: U_{x} S^{3} \rightarrow U S^{3}$ and get a special class of oriented spheres $\left(x, i_{x}\right): S^{2} \rightarrow U S^{3}, x \in S^{3}$, called the point spheres in $U S^{3}$. The so called Lie transformations are the diffeomorphisms from $U S^{3}$ to itself that take oriented spheres to oriented spheres.

Any oriented sphere $k$ can be represented by the equation $m=\cos \theta x$ $-\sin \theta n,(x, n) \in U S^{3}$, for some $m \in S^{3}$ and $\theta \in[0, \pi)$, and $k^{\prime}=(m, \cos \theta, \sin \theta)$ is uniquely determined by $k$ up to signs.

Let $R^{6}$ be $\mathbb{R}^{6}$ equipped with the product $\langle$,$\rangle defined by$

$$
\langle x, x\rangle=x_{1}^{2}+x_{2}^{2}+x_{3}^{2}+x_{4}^{2}-x_{5}^{2}-x_{6}^{2},\left(x_{1}, x_{2}, \cdots, x_{6}\right) \in \mathbb{R}^{6},
$$

and $\mathbb{Q}$ be the quadric in $\mathbb{P}^{5}$ defined by $\mathbb{Q}=\left\{[x] \in \mathbb{P}^{5} \mid\langle x, x\rangle=0\right\}$. Then we have a mapping from the set of oriented spheres in $U S^{3}$ to $\mathbb{Q}$ given by $k \rightarrow\left[k^{\prime}\right]$. It is easy to see that this mapping is bijective. Moreover, two oriented spheres $k_{1}, k_{2}$ in $U S^{3}$ are (oriented) contact if and only if 


$$
\left\langle k_{1}^{\prime}, k_{2}^{\prime}\right\rangle=0 .
$$

Thus we can identify the oriented spheres in $U S^{3}$ with the points in $\mathbb{Q}$. Since the point sphere $U_{x} S^{3}$ corresponds to the point $[x, 1,0]$ in $\mathbb{Q}$, we call such points in $\mathbb{Q}$ also point spheres.

\subsection{Lie diffeomorphism}

Any point $(x, n) \in U S^{3}$ determines uniquely one pencil of oriented spheres contacting each other at $x \in S^{3}$ with the same orientation $n$, which by (1.2) is the projective line on $\mathbb{Q}$ spanned by the points $[x, 1,0]$ and $[n, 0,1]$ in $\mathbb{Q}$. Thus we have a bijective mapping

$$
L: U S^{3} \longrightarrow \Lambda \text {, }
$$

where $A$ is the set consisting of all projective lines on $\mathbb{Q}$. Thus $A$ has the unique differential structure such that $L$ is a diffeomorphism. We call $L$ Lie diffeomorphism.

Let $O(4,2)$ be the orthogonal group preserving the inner product $\langle$, in (1.1). Then $O(4,2)$ is a transformation group of $\mathbb{Q}$ defined by

$$
A([x])=[x A], \quad \forall[x] \in \mathbb{Q}, \quad A \in O(4,2),
$$

where $x A$ is the product of two matrices $x$ and $A$. Since any element of $O(4,2)$ carries projective lines on $\mathbb{Q}$ to projective lines on $\mathbb{Q}, \mathbb{Q}(4,2)=$ $O(4,2) \bmod ( \pm 1)$ is naturally a transformation group of $\Lambda$. By a theorem of Pinkall in [9] we know that the mapping $\operatorname{Ad}(L): \phi \rightarrow L \phi L^{-1}$ is an isomorphism from Lie transformation group of $U S^{3}$ to $\mathbb{O}(4,2)$ of $\Lambda$. We call $\mathcal{O}(4,2)$ the Lie transformation group of $\Lambda$.

\subsection{Legendre surfaces, curvature spheres and curvature vectors}

Let $\lambda: M \rightarrow \Lambda$ be an immersion of surface. We write $L^{-1} \circ \lambda=(n, x): M$ $\rightarrow U S^{3}$. Then $\lambda$ is called a Legendre surface if $d x \cdot n=0$.

Let $k_{1}, k_{2} \in C^{\infty}(M)$ with $\left(k_{1}, k_{2}\right) \neq 0$ and $k=k_{1}(x, 1,0)+k_{2}(n, 0,1) . \quad[k]: M$ $\rightarrow \mathbb{Q}$ is called a curvature sphere of Legendre surface $\lambda$ if there is non-zero vector field $X \in T M$ such that

$$
[d k(X)(m)] \in \lambda(m) \subset \mathbb{Q}
$$

at any point $m \in M$. Such $X$ in (1.5) is called a curvature vector (field) corresponding to the curvature sphere [k]. By a theorem of Pinkall ([9], p. 433) we know that there are at most two curvature spheres $[a],[b]: M$ $\rightarrow \mathbb{Q}$. Moreover, if $\lambda$ is umbilic point free, i.e., $[a] \neq[b]$ on $M$, then the 
curvature vectors $E_{1}$ and $E_{2}$ (corresponding to $[a]$ and $[b]$ respectively) form a global basis for $T M$.

Let $\lambda: M \rightarrow \Lambda$ be a Legendre surface without umbilic point. We give an order for the curvature spheres $([a],[b])$ of $\lambda$. We call $(a, b): M \rightarrow R^{6}$ a curvature sphere representation of $([a],[b])$. Note that for any curvature sphere representation $(a, b)$ we have

$$
\begin{aligned}
& \langle a, a\rangle=\langle a, b\rangle=\langle b, b\rangle=0,\langle d a, b\rangle=-\langle a, d b\rangle=0, \\
& \lambda=\operatorname{span}(a, b): M \longrightarrow \Lambda,
\end{aligned}
$$

where $\operatorname{span}(a, b)(m)$ is the projective line on $\mathbb{Q}$ spanned by $[a(m)]$ and $[b(m)]$.

Let $E_{1}, E_{2}$ be the curvature vectors corresponding to $[a],[b]$ respectively. By definition we have $E_{1}(a), E_{2}(b) \in \operatorname{span}(a, b)$. Because of the signature of $\langle$,$\rangle we must have \left\langle E_{1}(b), E_{1}(b)\right\rangle>0$ and $\left\langle E_{2}(a), E_{2}(a)\right\rangle>0$ (cf. Pinkall [8], p. 92). We call $\left(E_{1}, E_{2}\right)$ unit curvature vector for the curvature sphere representation $(a, b)$ if

$$
\left\langle E_{1}(b), E_{1}(b)\right\rangle=\left\langle E_{2}(a), E_{2}(a)\right\rangle=1 .
$$

It is clear that the unit curvature vector $\left(E_{1}, E_{2}\right)$ is determined by $(a, b)$ up to signs.

\subsection{Lie sphere geometry}

In Lie sphere geometry we study the invariants of immersed surface $f=(x, h): M \rightarrow U S^{3}$ with $d x \cdot n=0$ under the Lie sphere transformation group of $U S^{3}$. By the above discussion we know that this geometry is equivalent to the geometry of Legendre surface $\lambda=L \circ f: M \rightarrow \Lambda$ under the transformation group $\mathbb{Q}(4,2)$ of $\Lambda$.

For any Legendre surface $\lambda$ we can construct a moving frame in $R^{6}$ along $M$ by using the curvature sphere representation and its unit curvature vectors. Since $O(4,2)$ or $O(4,2)$ is linear, we can use the same method as we do in euclidean geometry or affine geometry to give the Fundamental theorems for Lie geometry surface. In this paper we will use this idea to give the surface theory for Möbius differential geometry.

\section{§2. Möbius surfaces and Möbius transformations}

In this section we introduce some basic concepts in Möbius geometry in terms of the concepts in Lie sphere geometry.

Let $G_{3}$ be the Möbius transformation group of $S^{3}$. We identify $\sigma \in G_{3}$ 
with the diffeomorphism $\sigma^{\prime}: U S^{3} \rightarrow U S^{3}$ defined by

$$
\sigma^{\prime}(x, n)=\left(\sigma(x), \frac{d \sigma(n)}{|d \sigma|}\right) .
$$

Since $\sigma^{\prime}$ carries oriented spheres to oriented spheres, it is a Lie transformation. Thus $G_{3}$ can be regarded as a subgroup of the Lie transformation group of $U S^{3}$, and then $M G_{3}=L G_{3} L^{-1}$ is a subgroup of Lie transformation group $\mathrm{O}(4,2)$ of $\Lambda$, where $L$ is the Lie diffeomorphism.

Proposition 2.1. If a Lie transformation $B \in \mathbb{O}(4,2)$ takes point spheres in $\mathbb{Q}$ to point spheres, then $B=\left(\begin{array}{cc}A & 0 \\ 0 & 1\end{array}\right)$ and $A \in O(4,1)$, where $O(4,1)$ is the orthogonal group of $\mathbb{R}^{5}$ preserving the inner product $\langle w, w\rangle=w_{1}^{2}+w_{2}^{2}$ $+w_{3}^{2}+w_{4}^{2}-w_{5}^{2},\left(w_{1}, \cdots, w_{5}\right) \in \mathbb{R}^{5}$.

Proof. Let $B \in \mathbb{O}(4,2)$ take point spheres to point spheres. We write $B=\left(\begin{array}{ll}A & b \\ c & \varepsilon\end{array}\right)$ such that $A$ is a $5 \times 5$ matrix. Then for any $x \in S^{3} B([x, 1,0])$ $=[(x, 1) A,(x, 1) b]$ is a point sphere in $\mathbb{Q}$. Thus $(x, 1) b=0$ for all $x \in S^{3}$, so $b=0$. Since $B \in O(4,2)$ means exactly that $B I_{2}{ }^{t} B=I_{2}$, where $I_{2}=$ $\left(\begin{array}{rrr}I & 0 & 0 \\ 0 & -1 & 0 \\ 0 & 0 & -1\end{array}\right)=\left(\begin{array}{rr}I_{1} & 0 \\ 0 & -1\end{array}\right)$, we know that $A I_{1}^{t} A-b^{t} b=0, A I_{1}{ }^{t} c-\varepsilon b=0$ and $c I_{1}^{t} c-\varepsilon^{2}=-1$. From $b=0$ we obtain $c=0, \varepsilon= \pm 1$ and $A I_{1}{ }^{t} A$ $=I_{1}$. Therefore $B=\varepsilon\left(\begin{array}{cc}\varepsilon A & 0 \\ 0 & 1\end{array}\right)=\left(\begin{array}{cc}\varepsilon A & 0 \\ 0 & 1\end{array}\right)$ in $O(4,2)$ and $\varepsilon A \in O(4,1)$. Q.E.D.

Since any Möbius transformation $\sigma=\sigma^{\prime} \in G_{3}$ carries point spheres into point spheres, we know that $L \sigma^{\prime} L^{-1}=\left(\begin{array}{cc}A & 0 \\ 0 & 1\end{array}\right)$ for some $A \in O(4,1)$. Conversely, given $A \in O(4,1)$ we can define a Möbius transformation $\sigma: S^{3} \rightarrow S^{3}$ by $\sigma(x)=(x B+u) /(x v+w), x \in S^{3}$, where $\left(\begin{array}{ll}B & v \\ u & w\end{array}\right)=A$ and $B$ is a $4 \times 4$ matrix. One can easily verify that $L \sigma^{\prime} L^{-1}=\left(\begin{array}{cc}A & 0 \\ 0 & 1\end{array}\right)$. This gives a easy proof of the following well-known theorem:

Theorem 2.2. $M G_{3}=\left\{\left(\begin{array}{cc}A & 0 \\ 0 & 1\end{array}\right) \mid A \in O(4,1)\right\} \subset \mathbb{O}(4,2)$.

Now we want to represent the immersed surfaces in $S^{3}$ by a special class of Legendre surfaces in $A$.

Let $x: M \rightarrow S^{3}$ be an oriented surface without umbilic point and $n$ the unit normal of $x$ which gives the orientation. Let $k, h$ be the principal curvatures for $x$. Then $\lambda=L \circ(x, n): M \rightarrow \Lambda$ is a Legendre surface, and $(a, b)$ define by

$$
a=k(x, 1,0)+(n, 0,1), \quad b=h(x, 1,0)+(n, 0,1)
$$


is a curvature sphere representation for $\lambda$. The unit curvature vectors $E_{1}, E_{2}$ corresponding to the curvature spheres $([a],[b])$ are the principal directions of $x$ with euclidean length $(k-h)^{-1}$. Let $p: \mathbb{R}^{6} \rightarrow \mathbb{R}$ be the projection defined by

$$
p\left(x_{1}, x_{2}, \cdots, x_{6}\right)=x_{6}, \quad\left(x_{1}, x_{2}, \cdots, x_{6}\right) \in \mathbb{R}^{6} .
$$

By (2.2) we have $p(a)=p(b)=1$. This motivates

Definition 2.3. A Legendre surface $\lambda: M \rightarrow \Lambda$ is called a Möbius surface if (i) $\lambda$ is umbilic point free; and (ii) there exists a curvature sphere representation $(a, b)$ such that $p(a)=p(b)=1$.

Note that such $(a, b)$ is uniquely determined by the order of curvature spheres of $\lambda$, and the corresponding unit curvature vector $\left(E_{1}, E_{2}\right)$ is uniquely determined by (1.8) up to signs. We call $(a, b)$ the curvature sphere representation and $\left(E_{1}, E_{2}\right)$ the unit curvature vector for Möbius surface $\lambda$.

Definition 2.4. Let $\lambda: M \rightarrow \Lambda$ and $\mu: N \rightarrow \Lambda$ be Möbius surfaces. $\lambda$ and $\mu$ are said to be Möbius equivalent if there are a diffeomorphism $e: M \rightarrow N$ and $A \in M G_{3}$ such that $\mu \circ e=A \circ \lambda$. Such $\epsilon, A$ or $(e, A)$ is called a Möbius equivalence of $\lambda$ and $\mu$. Briefly, $\lambda$ and $\mu$ are Möbius equivalent if their images in $\Lambda$ differ only by a Möbius transformation.

By Theorem 2.2 we can easy see that

Proposition 2.5. The curvature sphere representation $(a, b)$ and the unit curvature vector $\left(E_{1}, E_{2}\right) \bmod ( \pm 1)$ are invariant under the Möbius transformation group $M G_{3}$.

We know from (2.2) that any oriented surface $x: M \rightarrow S^{3}$ without umbilic point defines a Möbius surface $\lambda=L \circ(x, n): M \rightarrow \Lambda$. Conversely we have

Theorem 2.6. Any Möbius surface $\lambda: M \rightarrow \Lambda$ is defined in this way by an oriented surface $x: M \rightarrow S^{3}$.

To prove this theorem we need the following proposition:

Proposition 2.7. Let $(a, b)$ be the curvature sphere representation for a Möbius surface $\lambda$ and $\left(E_{1}, E_{2}\right)$ its unit curvature vector. Then we have the following product table: 


\begin{tabular}{c|cccc}
$\langle\rangle$, & $a$ & $b$ & $E_{2}(a)$ & $E_{1}(b)$ \\
\hline$a$ & 0 & 0 & 0 & 0 \\
$b$ & 0 & 0 & 0 & 0 \\
$E_{2}(a)$ & 0 & 0 & 1 & 0 \\
$E_{1}(b)$ & 0 & 0 & 0 & 1
\end{tabular}

Proof. By (1.5) and (1.7) we have

$$
\left[E_{1}(a)\right],\left[E_{2}(b)\right] \in \operatorname{span}(a, b) .
$$

Thus (1.6) and (2.5) imply

$$
\left\langle E_{i}(a), b\right\rangle=-\left\langle a, E_{i}(b)\right\rangle=0, \quad i=1,2 .
$$

To prove Proposition 2.7 it suffices to show that $\left\langle E_{1}(b), E_{2}(a)\right\rangle=0$. Let $f, g$ be the smooth functions on $M$ such that $E_{1} E_{2}-E_{2} E_{1}=\left[E_{1}, E_{2}\right]=f E_{1}$ $+g E_{2}$, then we get from (2.5) that

$$
E_{1} E_{2}(a)=C a+C^{\prime} b+C^{\prime \prime} E_{2}(a), \quad C, C^{\prime}, C^{\prime \prime} \in C^{\infty}(M) .
$$

Thus we have $\left\langle E_{1}(b), E_{2}(a)\right\rangle=-\left\langle b, E_{1} E_{2}(a)\right\rangle=0$.

Q.E.D.

The proof of Theorem 2.6. Let $(a, b)$ and $\left(E_{1}, E_{2}\right)$ be as in Proposition 2.7. Let $\left(\theta_{1}, \theta_{2}\right)$ be the dual basis for $\left(E_{1}, E_{2}\right)$. We write $L^{-1} \circ \lambda=(x, n)$, $a=\left(a^{\prime}, k, 1\right)$ and $b=\left(b^{\prime}, h, 1\right)$. Then we have

$$
(x, 1,0)=\frac{a-b}{k-h}, \quad(n, 1,0)=\frac{k a-h b}{k-h} .
$$

From (2.4) and (2.8) we know that $x, n: M \rightarrow S^{3}, d x \cdot n=0$ and

$$
(d x, 0,0)=\omega a+\omega^{\prime} b+\frac{1}{k-h}\left(-E_{1}(b) \theta_{1}+E_{2}(a) \theta_{2}\right), \quad \omega, \omega^{\prime} \in T^{*} M .
$$

Now if $V \in T_{m} M$ such that $d x(V)=0$, we get from (2.9) and (2.4) that $\theta_{1}(V)=\theta_{2}(V)=0$. Thus $V=0$. So $x: M \rightarrow S^{3}$ is an immersion. Q.E.D.

COROLlary 2.8. The Möbius geometry of immersed surfaces without umbilic point in $S^{3}$ is equivalent by the Lie diffeomorphism to the geometry of Möbius surfaces in $\Lambda$ under the Möbius transformation group $M G_{3}$ for $\Lambda$.

\section{§ 3. Möbius invariants for Möbius surfaces}

In this section we give the Möbius invariant system for Möbius sur- 
faces and the relations among these invariants.

Let $\lambda: M \rightarrow \Lambda$ be a Möbius surface, $(a, b)$ the curvature sphere representation of $\lambda$ and $\left(E_{1}, E_{2}\right)$ its unit curvature vector. Let $\left(\theta_{1}, \theta_{2}\right)$ be the dual basis for $\left(E_{1}, E_{2}\right)$. We know by Proposition 2.5 that $(a, b),\left(E_{1}, E_{2}\right)$ $\bmod ( \pm 1)$ and $\left(\theta_{1}, \theta_{2}\right) \bmod ( \pm 1)$ are Möbius invariants. In particular we have the Möbius invariant metric

$$
d s^{2}=\langle d a, d a\rangle+\langle d b, d b\rangle=\theta_{1}^{2}+\theta_{2}^{2}
$$

on $M$. It is the so-called Möbius metric, whose volume functional is 4 times the Willmore functional in $S^{3}$.

The other two important Möbius invariants are the functions

$$
\Phi=\left\langle E_{2} E_{2}(a), E_{2} E_{2}(a)\right\rangle, \quad \Psi=\left\langle E_{1} E_{1}(b), E_{1} E_{1}(b)\right\rangle .
$$

We will show that $\Phi+\Psi=1$ and $\left(E_{1}, E_{2}, \Phi\right)$ forms a complete Möbius invariant system for Möbius surfaces.

First we come to list the relations among the Möbius invariants $E_{1}, E_{2}, \Phi, \Psi$. Let $f, g$ be smooth functions on $M$ such that

$$
d \theta_{1}=f \theta_{1} \wedge \theta_{2}, \quad d \theta_{2}=g \theta_{1} \wedge \theta_{2} .
$$

We denote $u_{\imath}=E_{i}(u), i=1,2$. Then $d u=\Sigma_{i} u_{i} \theta_{i}$. By exterior differential we get

$$
u_{12}=u_{21}+f u_{1}+g u_{2} .
$$

From (2.5) and the fact $p(a)=p(b)=1$ we know that

$$
a_{1}=S a-S b, \quad b_{2}=-T a+T b
$$

for some smooth functions $S$ and $T$ on $M$. Thus (3.4) and (3.5) imply

$$
\begin{aligned}
& a_{21}=\left(S_{2}+S T-f S\right) a-\left(S_{2}+S T-f S\right) b+(S-g) a_{2}, \\
& b_{12}=-\left(T_{1}+S T+g T\right) a+\left(T_{1}+S T+g T\right) b+(T+f) b_{1} .
\end{aligned}
$$

It follows from (2.4) that $f=-T$ and $g=S$. Therefore, (3.3) (3.7) can be simplified as

$$
\begin{aligned}
& d \theta_{1}=-T \theta_{1} \wedge \theta_{2}, \quad d \theta_{2}=S \theta_{1} \wedge \theta_{2} . \\
& u_{12}=u_{21}-T u_{1}+S u_{2}, \quad \text { i.e., }\left[E_{1}, E_{2}\right]=T E_{1}-S E_{2} . \\
& a_{21}=\left(S_{2}+2 S T\right) a-\left(S_{2}+2 S T\right) b . \\
& b_{12}=-\left(T_{1}+2 S T\right) a+\left(T_{1}+2 S T\right) b .
\end{aligned}
$$

From $(3.4)^{\prime}$ we know that $S, T$ are determined by $\left(E_{1}, E_{2}\right)$. 
Now we define

$$
c=a_{22}+\frac{1}{2} \Phi a, \quad d=b_{11}+\frac{1}{2} \Psi b,
$$

where $\Phi$ and $\Psi$ are defined by (3.2). Using $(3.4)^{\prime},(3.6)^{\prime}$ and (3.5) we obtain

$$
\begin{aligned}
c_{1}= & a_{221}+\frac{1}{2} \Phi_{1} a+\frac{1}{2} \Phi a_{1} \\
= & a_{212}+T a_{21}-S a_{22}+\frac{1}{2} \Phi_{1} a+\frac{1}{2} \Phi(S a-S b) \\
= & \left(S_{22}+4 S_{2} T+2 S T_{2}+4 S T^{2}+\Phi S+\frac{1}{2} \Phi_{1}\right) a \\
& -\left(S_{22}+4 S_{2} T+2 S T_{2}+4 S T^{2}+\frac{1}{2} \Phi S\right) b+\left(S_{2}+2 S T\right) a_{2}-S c .
\end{aligned}
$$

Similarly from $(3.4)^{\prime},(3.7)^{\prime}$ and (3.5) we get

$$
\begin{aligned}
d_{2}=-( & \left.T_{11}+2 S_{1} T+4 S T_{1}+4 S^{2} T+\frac{1}{2} \Psi T\right) a \\
& +\left(T_{11}+2 S_{1} T+4 S T_{1}+4 S^{2} T+\Psi T+\frac{1}{2} \Psi_{2}\right) b \\
& +\left(T_{1}+2 S T\right) b_{1}-T d .
\end{aligned}
$$

Since by (3.8) we have

$$
\begin{array}{ll}
\langle c, c\rangle=\langle d, d\rangle=0, & \langle c, a\rangle=\langle d, b\rangle=-1, \\
\left\langle c, a_{2}\right\rangle=\left\langle d, b_{1}\right\rangle=0, & \langle c, b\rangle=\langle d, a\rangle=0,
\end{array}
$$

so (2.4), (3.9) and (3.10) imply that

$$
\begin{aligned}
& S_{22}+4 S_{2} T+2 S T_{2}+4 S T^{2}+\Phi S+\frac{1}{2} \Phi_{1}=0 \\
& T_{11}+4 S T_{1}+2 S_{1} T+4 S^{2} T+\Psi T+\frac{1}{2} \Psi_{2}=0
\end{aligned}
$$

They are two relations among the Möbius invariants $\left(E_{1}, E_{2}, \Phi, \Psi\right)$. Thus (3.9) and (3.10) become

$$
\begin{aligned}
& c_{1}=\frac{1}{2}\left(\Phi S+\Phi_{1}\right) b+\left(S_{2}+2 S T\right) a_{2}-S c \\
& d_{2}=\frac{1}{2}\left(\Psi T+\Psi_{2}\right) a+\left(T_{1}+2 S T\right) b_{1}-T d
\end{aligned}
$$


It follows that $\langle c, d\rangle=\left\langle c, b_{11}\right\rangle=\left(\left\langle c, b_{1}\right\rangle\right)_{1}-\left\langle c_{1}, b_{1}\right\rangle=-\left(\left\langle a_{2}, b_{12}\right\rangle\right)_{1}=0$. Thus from (2.4) and (3.11) we obtain the following product table:

\begin{tabular}{l|rrrrrr}
$\langle\rangle$, & $a$ & $b$ & $a_{2}$ & $b_{1}$ & $c$ & $d$ \\
\hline$a$ & 0 & 0 & 0 & 0 & -1 & 0 \\
$b$ & 0 & 0 & 0 & 0 & 0 & -1 \\
$a_{2}$ & 0 & 0 & 1 & 0 & 0 & 0 \\
$b_{1}$ & 0 & 0 & 0 & 1 & 0 & 0 \\
$c$ & -1 & 0 & 0 & 0 & 0 & 0 \\
$d$ & 0 & -1 & 0 & 0 & 0 & 0
\end{tabular}

A basis $\left(a, b, a_{2}, b_{1}, c, d\right)$ for $R^{6}$ satisfying this product table is called skew Lie basis. A Lie basis for $R^{6}$ is a basis $\left(e_{1}, e_{2}, \cdots, \epsilon_{6}\right)$ such that the matrix

$$
\left(\left\langle e_{i}, e_{j}\right\rangle\right)_{1 \leq i, j \leq 6}=I_{2}=\left(\begin{array}{rrr}
I & 0 & 0 \\
0 & -1 & 0 \\
0 & 0 & -1
\end{array}\right) .
$$

Proposition 3.1. Let $\left(a, b, a_{2}, b_{1}, c, d\right)$ and $\left(a^{\prime}, b^{\prime}, a_{2}^{\prime}, b_{1}^{\prime}, c^{\prime}, d^{\prime}\right)$ be two skew Lie basis for $R^{6}$. Then there exists unique $A \in O(4,2)$ such that $\left(a^{\prime}, b^{\prime}, a_{2}^{\prime}, b_{1}^{\prime}, c^{\prime}, d^{\prime}\right)=\left(a A, b A, a_{2} A, b_{1} A, c A, d A\right)$.

Proposition 3.2. A basis $\left(a, b, a_{2}, b_{1}, c, d\right)$ for $R^{6}$ is a skew Lie basis if and only if $\left(\frac{1}{\sqrt{2}}(a-c), \frac{1}{\sqrt{2}}(b-d), a_{2}, b_{1}, \frac{1}{\sqrt{2}}(a+c), \frac{1}{\sqrt{2}}(b+d)\right)$ is a Lie basis. $O(4,2)$.

Proposition 3.3. $\left(e_{1}, e_{2}, \cdots, e_{6}\right)$ is a Lie basis if and only if $\left(\begin{array}{c}e_{1} \\ e_{2} \\ \vdots \\ e_{6}\end{array}\right) \epsilon$

Proposition 3.4. $A \in O(4,2)$ if and only if ${ }^{t} A \in O(4,2)$.

The proof of these propositions is left to the reader. For any Möbius surface $\lambda: M \rightarrow \Lambda$ (or equivalently immersed surface $x: M \rightarrow S^{3}$ ) we have defined a moving skew Lie frame $\left(a, b, a_{2}, b_{1}, c, d\right)$ in $R^{6}$ along $M$. Thus

$$
\left(e_{1}, e_{2}, \cdots, e_{6}\right)=\left(\frac{1}{\sqrt{2}}(a-c), \frac{1}{\sqrt{2}}(b-d), a_{2}, b_{1}, \frac{1}{\sqrt{2}}(a+c), \frac{1}{\sqrt{2}}(b+d)\right)
$$


is a Lie frame along $M$. By Propositions 3.3 and 3.4 we know that $A=\left(\begin{array}{c}e_{1} \\ e_{2} \\ \vdots \\ e_{6}\end{array}\right): M \rightarrow O(4,2)$ and ${ }^{t} A=\left(\begin{array}{c}e_{1}^{*} \\ e_{2}^{*} \\ \vdots \\ e_{6}^{*}\end{array}\right): M \rightarrow O(4,2)$. Since $p(a)=p(b)=1$, $p(c)=\frac{1}{2} \Phi$ and $p(d)=\frac{1}{2} \Psi$ (see (3.8)), then $e_{6}^{*}=\left(p\left(e_{1}\right), p\left(e_{2}\right), \cdots, p\left(e_{6}\right)\right)=$ $\left(\frac{1}{\sqrt{2}}\left(1-\frac{1}{2} \Phi\right), \frac{1}{\sqrt{2}}\left(1-\frac{1}{2} \Psi\right), 0,0, \frac{1}{\sqrt{2}}\left(1+\frac{1}{2} \Phi\right), \frac{1}{\sqrt{2}}\left(1+\frac{1}{2} \Psi\right)\right)$. Thus from $\left\langle e_{6}^{*}, e_{6}^{*}\right\rangle=-1$ we obtain

$$
\Phi+\Psi=1 .
$$

Now we compare the Möbius invariants for the immersed surface $x: M \rightarrow S^{3}$ with the euclidean invariants. Let $k, h$ be the two principal curvatures for $x$ corresponding to the unit curvature vector fields $e_{1}, e_{2}$ respectively. We denote by $u_{i}$ the directional derivative $e_{i}(u)$ and $d x^{2}$ the induced euclidean metric for $x$. Then we have

$$
\begin{aligned}
E_{1} & =\frac{e_{1}}{k-h}, \quad E_{2}=\frac{e_{2}}{k-h} \\
d s^{2} & =(k-h)^{2} d x^{2} \\
S & =\frac{k_{1}}{(k-h)^{2}}, \quad T=\frac{h_{2}}{(k-h)^{2}} \\
\Phi & =\frac{h_{1}^{2}}{(k-h)^{4}}+\frac{k_{2}^{2}}{(k-h)^{4}}+\frac{h^{2}+1}{(k-h)^{2}}-\frac{2}{(k-h)^{2}}\left(\frac{k_{2}}{k-h}\right)_{2} \\
\Psi & =\frac{h_{1}^{2}}{(k-h)^{4}}+\frac{k_{2}^{2}}{(k-h)^{4}}+\frac{k^{2}+1}{(k-h)^{2}}+\frac{2}{(k-h)^{2}}\left(\frac{h_{1}}{k-h}\right)_{1}
\end{aligned}
$$

Let $V$ be the area functional with respect to the Möbius metric. The critical points of $V$ are called the Möbius minimal surfaces or the Willmore surfaces, which can be defined by the Euler-Lagrange equation

$$
2\left(S_{1}-T_{2}\right)+4\left(S^{2}-T^{2}\right)+1-2 \Phi=0
$$

where $S_{1}=E_{1}(S)$ and $T_{2}=E_{2}(T)$. We will see in the last section that the Clifford torus is the Möbius minimal surface corresponding to the Möbius invariants $S=T=0$ and $\Phi=1 / 2$.

\section{§4. Fundamental theorems for the Möbius surfaces}

For any Möbius surface we have defined in $\S 3$ a moving skew Lie 
frame $\left(a, b, a_{2}, b_{1}, c, d\right)$ along the surface. In this section we use the theory of Partial Differential Equations (PDE) to establish the fundamental theorems for Möbius surfaces. For this purpose we need to calculate the differentials of $\left(a, b, a_{2}, b_{1}, c, d\right)$.

By (3.14) we know that for any $u: M \rightarrow R^{6}$ we have the formula

$$
u=-\langle u, c\rangle a-\langle u, d\rangle b+\left\langle u, a_{2}\right\rangle a_{2}+\left\langle u, b_{1}\right\rangle b_{1}-\langle u, a\rangle c-\langle u, b\rangle d .
$$

Using (3.14), (3.10)', (3.7) $)^{\prime}$ and (3.5) we obtain

$$
\begin{aligned}
& \left\langle c_{2}, c\right\rangle=0,\left\langle c_{2}, d\right\rangle=-\left\langle c, d_{2}\right\rangle=\frac{1}{2}\left(\Psi T+\Psi_{2}\right), \\
& \left\langle c_{2}, a_{2}\right\rangle=-\left\langle c, a_{22}\right\rangle=-\frac{1}{2} \Phi, \quad\left\langle c_{2}, b_{1}\right\rangle=-\left\langle c, b_{12}\right\rangle=-\left(T_{1}+2 S T\right), \\
& \left\langle c_{2}, a\right\rangle=-\left\langle c, a_{2}\right\rangle=0, \quad\left\langle c_{2}, b\right\rangle=-\left\langle c, b_{2}\right\rangle=-T,
\end{aligned}
$$

which imply that

$$
c_{2}=-\frac{1}{2}\left(\Psi T+\Psi_{2}\right) b-\frac{1}{2} \Phi a_{2}-\left(T_{1}+2 S T\right) b_{1}+T d .
$$

Similarly from (3.14), $(3.9)^{\prime},(3.6)^{\prime}$ and (3.5) we get

$$
d_{1}=-\frac{1}{2}\left(\Phi S+\Phi_{1}\right) a-\left(S_{2}+2 S T\right) a_{2}-\frac{1}{2} \Psi b_{1}+S c .
$$

So we have the following structure equations for the Möbius surface $\lambda: M \rightarrow \Lambda:$

$$
\left\{\begin{array}{l}
E_{1}(a)=S a-S b, \quad E_{2}(a)=a_{2}, \\
E_{1}(b)=b_{1}, \quad E_{2}(b)=-T a+T b, \\
E_{1}\left(a_{2}\right)=\left(S_{2}+2 S T\right) a-\left(S_{2}+2 S T\right) b, \quad E_{2}\left(a_{2}\right)=c-\frac{1}{2} \Phi a, \\
E_{1}\left(b_{1}\right)=d-\frac{1}{2}(1-\Phi) b, \quad E_{2}\left(b_{1}\right)=-\left(T_{1}+2 S T\right) a+\left(T_{1}+2 S T\right) b, \\
E_{1}(c)=\frac{1}{2}(\Phi S+\Phi) b+\left(S_{2}+2 S T\right) a_{2}-S c, \\
E_{2}(c)=-\frac{1}{2}\left(T-\Phi T-\Phi_{2}\right) b-\frac{1}{2} \Phi a_{2}-\left(T_{1}+2 S T\right) b_{1}+T d, \\
E_{1}(d)=-\frac{1}{2}(\Phi S+\Phi) a-\left(S_{2}+2 S T\right) a_{2}-\frac{1}{2}(1-\Phi) b_{1}+S c, \\
E_{2}(d)=\frac{1}{2}\left(T-\Phi T-\Phi_{2}\right) a+\left(T_{1}+2 S T\right) b_{1}-T d,
\end{array}\right.
$$


where $S, T$ are defined by

$$
\left[E_{1}, E_{2}\right]=T E_{1}-S E_{2} .
$$

Therefore, the structure equations (4.4) is completely determined by the Möbius invariants $\left(E_{1}, E_{2}, \Phi\right)$. By (3.12), (3.13) and (3.16) we have the following relations among $\left(E_{1}, E_{2}, \Phi\right)$ :

$$
\begin{aligned}
& S_{22}+4 S_{2} T+2 S T_{2}+4 S T^{2}+\Phi S+\frac{1}{2} \Phi_{1}=0 \\
& T_{11}+4 S T_{1}+2 S_{1} T+4 S^{2} T+(1-\Phi) T-\frac{1}{2} \Phi_{2}=0 .
\end{aligned}
$$

Proposition 4.1. (4.6) and (4.7) are exactly the integrability conditions for PDE (4.4).

Proof. As well-known the integrability conditions for (4.4) are given by the equations

$$
E_{1} E_{2}\left(x^{i}\right)=E_{2} E_{1}\left(x^{i}\right)+T E_{1}\left(x^{i}\right)-S E_{2}\left(x^{i}\right), \quad 1 \leq i \leq 6,
$$

(cf. (4.5)), where $\left(x^{1}, x^{2}, \cdots, x^{6}\right)=\left(a, b, a_{2}, b_{1}, c, d\right)$. It is straightforward to check from (4.4) that (4.8) is equivalent to (4.6) and (4.7). Q.E.D.

Now let $M$ be a simply connected surface, $\left(E_{1}, E_{2}\right)$ a basis for $T M$, $(S, T)$ the smooth functions defined by (4.5) and $\Phi \in C^{\infty}(M)$.

Definition 4.2. $\left(E_{1}, E_{2}, \Phi\right)$ is called a Möbius invariant system on $M$ if it satisfies (4.6) and (4.7). We denote by $Y(M)$ the space of all Möbius invariant systems on $M$.

For any $y=\left(E_{1}, E_{2}, \Phi\right) \in Y(M)$ we can define by (4.4) a linear PDE system. By the basic theory of $\mathrm{PDE}$ we know that given any point $m \in M$ and $I=\left(x_{0}^{1}, x_{0}^{2}, \cdots, x_{0}^{6}\right), x_{0}^{i} \in R^{6}$, there exists the unique solution $(a, b)$ (or equivalently $\left(a, b, a_{2}, b_{1}, c, d\right)$ ) for the PDE (4.4) with respect to $y$ such that

$$
\left(a, b, a_{2}, b_{1}, c, d\right)(m)=I .
$$

This solution is global because $M$ is simply connected.

Proposition 4.3. If $I=\left(x_{0}^{i}\right)=\left(x_{0}^{1}, x_{0}^{2}, \cdots, x_{0}^{6}\right)$ is a skew Lie basis for $R^{6}$, then the solution $\left(a, b, a_{2}, b_{1}, c, d\right)$ is a moving skew Lie frame along $M$.

Proof. We denote $\left(x^{i}\right)=\left(x^{1}, x^{2}, \cdots, x^{6}\right)=\left(a, b, a_{2}, b_{1}, c, d\right)$. Using (4.4) we can obtain a linear $\operatorname{PDE}$ for $\left\langle x^{i}, x^{j}\right\rangle, 1 \leq i, j \leq 6$, by calculating 
$E_{1}\left(\left\langle x^{i}, x^{j}\right\rangle\right)$ and $E_{2}\left(\left\langle x^{i}, x^{j}\right\rangle\right)$. It is straightforward to check that this PDE is also satisfied by $\left\langle x_{0}^{i}, x_{0}^{j}\right\rangle$ if $I=\left(x_{0}^{i}\right)$ is a skew Lie basis (cf. (3.14)). Since $\left\langle x^{i}, x^{j}\right\rangle(m)=\left\langle x_{0}^{i}, x_{0}^{j}\right\rangle$, by the uniqueness theorem of linear PDE we know that $\left\langle x^{i}, x^{j}\right\rangle \equiv\left\langle x_{0}^{i}, x_{0}^{j}\right\rangle$, i.e., $\left(a, b, a_{2}, b_{1}, c, d\right)$ is a moving skew Lie frame along $M$.

Q.E.D.

In particular, we have $\langle a, a\rangle=\langle a, b\rangle=\langle b, b\rangle=0$ and $\langle d a, b\rangle=0$. We define $\lambda_{y}(m, I)=\operatorname{span}(a, b): M \rightarrow \Lambda$. One can easily see that $\lambda_{y}(m, I)$ is a Legendre surface. But in general it is not a Möbius surface. In order to obtain by this way a Möbius surface we have to put more restriction on the initial value $(m, I)$.

Definition 4.4. Let $y=\left(E_{1}, E_{2}, \Phi\right) \in Y(M)$. A pair $(m, I)$ is called a Möbius initial value of $y$ if $m \in M$ and $I=\left(x_{0}^{i}\right)$ is a skew Lie basis for $R^{6}$ such that

$$
p(I)=\left(p\left(x_{0}^{1}\right), p\left(x_{0}^{2}\right), \cdots, p\left(x_{0}^{6}\right)\right)=\left(1,1,0,0, \frac{1}{2} \Phi(m), \frac{1}{2}(1-\Phi(m))\right) .
$$

Proposition 4.5. For any $y \in Y(M)$ there exists Möbius initial values.

Proof. Let $m \in M$, we define

$$
\begin{aligned}
e_{6}^{*}=\left(\frac{1}{\sqrt{2}}(1-\right. & \left.\frac{1}{2} \Phi(m)\right), \frac{1}{\sqrt{2}}\left(\frac{1}{2}+\frac{1}{2} \Phi(m)\right), 0,0 \\
& \left.\frac{1}{\sqrt{2}}\left(1+\frac{1}{2} \Phi(m)\right), \frac{1}{\sqrt{2}}\left(\frac{3}{2}-\frac{1}{2} \Phi(m)\right)\right),
\end{aligned}
$$

then $\left\langle e_{6}^{*}, e_{6}^{*}\right\rangle=-1$. We can extend $e_{6}^{*}$ to a Lie basis $\left(e_{1}^{*}, e_{2}^{*}, \cdots, e_{6}^{*}\right)$ for $R^{6}$. Since $A=\left(\begin{array}{c}e_{1}^{*} \\ e_{2}^{*} \\ \vdots \\ e_{6}^{*}\end{array}\right) \in O(4,2)$, then ${ }^{t} A=\left(\begin{array}{c}e_{1} \\ e_{2} \\ \vdots \\ e_{6}\end{array}\right) \in O(4,2)$ and $e_{6}^{*}=\left(p\left(e_{1}\right)\right.$, $\left.p\left(e_{2}\right), \cdots, p\left(e_{6}\right)\right)$. We define

$$
I=\left(\frac{1}{\sqrt{2}}\left(e_{1}+e_{5}\right), \frac{1}{\sqrt{2}}\left(e_{2}+e_{6}\right), e_{3}, e_{4}, \frac{1}{\sqrt{2}}\left(e_{5}-e_{1}\right), \frac{1}{\sqrt{2}}\left(e_{6}-e_{2}\right)\right),
$$

then $I$ is a skew Lie basis such that (4.10) holds. Thus $(m, I)$ is a Möbius initial value of $y$.

Q.E.D.

Proposition 4.6. If $(m, I)$ and $\left(m, I^{\prime}\right)$ are two Möbius initial values of $y \in Y(M)$, then there exists a Möbius transformation $B \in M G_{3}$ such that $I B=I^{\prime}$, where $I=\left(x^{1}, x^{2}, \cdots, x^{6}\right)$ and $I B=\left(x^{1} B, x^{2} B, \cdots, x^{6} B\right)$. 
Proof. Since $I$ and $I^{\prime}$ are skew Lie basis, we can find $B \in O(4,2)$ such that $I B=I^{\prime}$. We write $x^{i}=\left(x_{0}^{i}, p\left(x^{i}\right)\right), 1 \leq i \leq 6$, and $B=\left(\begin{array}{ll}A & v \\ u & w\end{array}\right)$, $w \in \mathbb{R}$. By (4.10) we have $p(I)=P\left(I^{\prime}\right)=P(I B)$, which implies that $x_{0}^{i} \cdot v$ $+(w-1) p\left(x^{i}\right)=0,1 \leq i \leq 6$. But $I=\left(x^{1}, x^{2}, \cdots, x^{6}\right)$ is a basis for $R^{6}$, we know that $v=0$ and $w=1$. Then $u=0$ follows from the fact that $B \in O(4,2)$, so we have $B \in M G_{3}$.

Q.E.D.

Now we can state the fundamental theorems for Möbius surfaces in $\Lambda$.

Theorem 4.7. Let $y=\left(E_{1}, E_{2}, \Phi\right) \in Y(M)$ be any Möbius invariant system on $M$. Let $(a, b)$ be the solution of (4.4) defined by $y$ and a Möbius initial value $(m, I)$ of $y$. Then $\lambda_{y}(m, I)=\operatorname{span}(a, b): M \rightarrow \Lambda$ is a Möbius surface with $(a, b)$ as its curvature sphere representation, $\left(E_{1}, E_{2}\right)$ as its unit curvature vector and $\Phi$ the Möbius invariant for $\lambda_{y}(m, I)$ defined by (3.2).

Theorem 4.8. The Möbius equivalent class $\lambda_{y}$ of $\lambda_{y}(m, I)$ is independent of the choices of the Möbius initial value $(m, I)$ of $y$.

Corollary 4.9. $\left(E_{1}, E_{2}, \Phi\right)$ is a complete Möbius invariant system for the Möbius surfaces in $\Lambda$ (umbilic-point-free immersed surfaces in $S^{3}$ ). These invariants are related by (4.6) and (4.7).

The proof of Theorem 4.7. From (4.4) we have $\left[E_{1}(a)\right] \in \operatorname{span}(a, b)$ and $\left[E_{2}(b)\right] \in \operatorname{span}(a, b)$, and by Proposition 4.3 we have $\left\langle a_{2}, a_{2}\right\rangle=\left\langle b_{1}, b_{1}\right\rangle=1$. So to prove the theorem it suffices to show that $p(a)=p(b)=1$. Since $\left(a, b, a_{2}, b_{1}, c, d\right)$ is the solution for PDE (4.4) with respect to $y$ and $(m, I)$, then $\left(p(a), p(b), p\left(a_{2}\right), p\left(b_{1}\right), p(c), p(d)\right)$ is the solution for PDE (4.4) with the initial value $(m, p(I))$. But we can directly verify from (4.4) that $\left(1,1,0,0, \frac{1}{2} \Phi, \frac{1}{2}(1-\Phi)\right)$ is also the solution of (4.4) with the same initial value $(m, p(I))$ (cf. (4.10)). By the uniqueness theorem of linear PDE system we have

$$
\left(p(a), p(b), p\left(a_{2}\right), p\left(b_{1}\right), p(c), p(d)\right)=\left(1,1,0,0, \frac{1}{2} \Phi, \frac{1}{2}(1-\Phi)\right)
$$

in particular, $p(a)=p(b)=1$.

Q.E.D.

The proof of Theorem 4.8. We have to show that if $\left(m^{\prime}, I^{\prime}\right)$ is another Möbius initial value of $y$, then $\lambda_{y}\left(m^{\prime}, I^{\prime}\right)$ is Möbius equivalent to $\lambda_{y}(m, I)$. Let $\left(a^{\prime}, b^{\prime}\right)$ be the solution of (4.4) with respect to $y$ and $\left(m^{\prime}, I^{\prime}\right)$, then by the same reason as (4.11) we have $\left(p\left(a^{\prime}\right), p\left(b^{\prime}\right), p\left(a_{2}^{\prime}\right), p\left(b_{1}^{\prime}\right), p\left(c^{\prime}\right), p\left(d^{\prime}\right)\right)=$ 
$\left(1,1,0,0, \frac{1}{2} \Phi, \frac{1}{2}(1-\Phi)\right)$. Thus the Möbius initial value $\left(m, I^{*}\right)$ of $y$, where $I^{*}=\left(p\left(a^{\prime}\right), p\left(b^{\prime}\right), p\left(a_{2}^{\prime}\right), p\left(b_{1}^{\prime}\right), p\left(c^{\prime}\right), p\left(d^{\prime}\right)\right)(m)$, satisfies $\lambda_{y}\left(m^{\prime}, I^{\prime}\right)=$ $\operatorname{span}\left(a^{\prime}, b^{\prime}\right)=\lambda_{y}\left(m, I^{*}\right)$. By Proposition 4.6 we can find $B \in M G_{3}$ such that $I B=I^{*}$. Thus $\left(a^{\prime}, b^{\prime}\right)$ and $(a B, b B)$ are two solutions for (4.4) with the same initial value $\left(m, I^{*}\right)=(m, I B)$. Therefore $\left(a^{\prime}, b^{\prime}\right)=(a B, b B)$ and $\lambda_{y}\left(m, I^{*}\right)=\operatorname{span}\left(a^{\prime}, b^{\prime}\right)=\operatorname{span}(a B, b B)=B \circ \lambda_{y}(m, I)$.

Q.E.D.

Let $\mathfrak{M} \ddot{o}(M)$ denote the set of Möbius equivalent classes of all Möbius surfaces from $M$ to $\Lambda$ (or $S^{3}$ ). By Theorems 4.7 and 4.8 we have a surjective mapping $\lambda: Y(M) \rightarrow \mathfrak{M} \ddot{\mathrm{o}}(M)$ defined by $\lambda(y)=\lambda_{y}$.

Proposition 4.10. Let $y=\left(E_{1}, E_{2}, \Phi\right) \in Y(M)$. Then (i) $y^{*}=\left(\varepsilon_{1} E_{1}, \varepsilon_{2} E_{2}\right.$, $\Phi) \in Y(M), \varepsilon_{i}= \pm 1$; (ii) $e^{*} y=\left(e_{*}^{-1} E_{1}, e_{*}^{-1} E_{2}, \Phi \circ e\right) \in Y(M)$ for any diffeomorphism $e: M \rightarrow M$; (iii) $\varepsilon(y)=\left(E_{2}, E_{1}, 1-\Phi\right) \in Y(M)$.

This proposition follows immediately from (4.6) and (4.7). Now we introduce a relation $\cong$ on $Y(M)$ by letting $\left(E_{1}, E_{2}, \Phi\right) \cong\left(E_{1}^{\prime}, E_{2}^{\prime}, \Phi^{\prime}\right)$ if there exists a diffeomorphism $e: M \rightarrow M$ such that $\left(E_{1}^{\prime}, E_{2}^{\prime}, \Phi^{\prime}\right)=\left(\varepsilon_{1} e_{*}^{-1} E_{1}, \varepsilon_{2} e_{*}^{-1} E_{2}\right.$, $\Phi \circ e), \varepsilon_{i}= \pm 1$. It is clear that $\cong$ is an equivalent relation in $Y(M)$.

TheOREM 4.11. $\lambda(y)=\lambda\left(y^{\prime}\right)$ in $\mathfrak{M} \ddot{o}(M)$ if and only if $y^{\prime} \cong y$ or $y^{\prime} \cong \varepsilon(y)$ in $Y(M)$.

Proof. Let $(a, b)$ be the solution for (4.4) with respect to $y$ and its Möbius initial value $(m, I)=\left(m, x_{0}^{i}\right)$. Then $(a, b)($ resp. $(a \circ e, b \circ e) ;(b, a))$ is the solution for (4.4) with respect to $y^{*}$ (resp. $\left.e^{*} y ; \varepsilon(y)\right)$ and its Möbius initial value $\left(m, I^{*}\right)\left(\operatorname{resp} .\left(e^{-1}(m), I\right) ;(m, \varepsilon(I))\right)$, where $I^{*}=\left(x_{0}^{1}, x_{0}^{2}, \varepsilon_{2} x_{0}^{3}, \varepsilon_{1} x_{0}^{4}, x_{0}^{5}, x_{0}^{6}\right)$ and $\varepsilon(I)=\left(x_{0}^{2}, x_{0}^{1}, x_{0}^{4}, x_{0}^{3}, x_{0}^{6}, x_{0}^{5}\right)$ (cf. Proposition 4.10). Thus we have $\lambda_{y^{*}}\left(m, I^{*}\right)$ $=\operatorname{span}(a, b)=\lambda_{y}(m, I), \quad \lambda_{e^{*} y}\left(e^{-1}(m), I\right)=\operatorname{span}(a \circ e, b \circ e)=\lambda_{y}(m, I) \circ e \quad$ and $\lambda_{\varepsilon(y)}(m, \varepsilon(I))=\operatorname{span}(b, a)=\operatorname{span}(a, b)=\lambda_{y}(m, I)$. Therefore, $\lambda(y)=\lambda\left(y^{\prime}\right)$ if $y^{\prime} \cong y$ or $y^{\prime} \cong \varepsilon(y)$. Conversely, if $y=\left(E_{1}, E_{2}, \Phi\right)$ and $y^{\prime}=\left(E_{1}^{\prime}, E_{2}^{\prime}, \Phi^{\prime}\right)$ in $Y(M)$ such that $\lambda(y)=\lambda\left(y^{\prime}\right)$, then we have a Möbius equivalence $(e, A)$ with $A \circ \lambda_{y}(m, I)=\lambda_{y^{\prime}}\left(m^{\prime}, I^{\prime}\right)$ ce , where $(m, I)$ and $\left(m^{\prime}, I^{\prime}\right)$ is the Möbius initial value of $y$ and $y^{\prime}$ respectively. Let $(a, b)$ (resp. $\left(a^{\prime}, b^{\prime}\right)$ ) be the solution for (4.4) with respect to $y$ and $(m, I)$ (resp. $y^{\prime}$ and $\left(m^{\prime}, I^{\prime}\right)$ ). Since both $\left(a^{\prime}, b^{\prime}\right)$ and $\left(a \circ e^{-1} A, b \circ e^{-1} A\right)$ are the curvature sphere representation for $\lambda_{y^{\prime}}\left(m^{\prime}, I^{\prime}\right)$, we have either (i) $\left(a^{\prime}, b^{\prime}\right)=\left(a \circ e^{-1} A, b \circ e^{-1} A\right)$ or (ii) $\left(b^{\prime}, a^{\prime}\right)$ $=\left(a \circ e^{-1} A, b \circ e^{-1} A\right)$. As $\left(E_{1}^{\prime}, E_{2}^{\prime}\right)$ and $\left(e_{*} E_{1}, e_{*} E_{2}\right)$ are the unit curvature vector for $\lambda_{y^{\prime}}\left(m^{\prime}, I^{\prime}\right)$, Case (i) implies $\left(E_{1}^{\prime}, E_{2}^{\prime}\right)=\left(\varepsilon_{1} e_{*} E_{1}, \varepsilon_{2} e_{*} E_{2}\right), \varepsilon_{i}= \pm 1$ and $\Phi^{\prime} \circ e=\Phi$; Case (ii) implies $\left(E_{2}^{\prime}, E_{1}^{\prime}\right)=\left(\varepsilon_{1} e_{*} E_{1}, \varepsilon_{2} e_{*} E_{2}\right)$ and $\Phi^{\prime} \circ e=1-\Phi$ 
(cf. (3.2)). Thus $y^{\prime} \cong y$ or $y^{\prime} \cong \varepsilon(y)$.

Q.E.D.

We know from Theorem 4.11 that $\lambda: Y(M) \rightarrow \mathfrak{M o}(M)$, induces a mapping $\lambda: Y(M) / \cong \rightarrow \mathfrak{M} \ddot{o}(M)$, which is a 2-sheet covering with the nontrivial covering transformation $\varepsilon$ induced by (iii) of Proposition 4.10.

\section{$\S 5$. Classification of Dupin surfaces in $E^{3}$ under the conformal transformation group}

A surface $M$ in $S^{3}$ or $E^{3}$ is called a Dupin surface if each principal curvature of $M$ is constant along its curvature leaves. As well known, the only Dupin surface in $E^{3}$ are spheres, planes and the so-called cyclides of Dupin.

The cyclides of Dupin are described in the book of Cecil and Ryan ([4], p. 151-166). These descriptions depend on the classical theorem: two focal surfaces for Dupin surfaces without umbilic point in $E^{3}$ are a pair of focal conics defined by Eisenhart ([5], p. 226).

Since Dupin surfaces are Möbius invariant, we can use the techniques in Möbius geometry to classify them under the Möbius transformation group. Let $x: M \rightarrow S^{3}$ be a surface without umbilic point. We may assume the $M$ is simply connected. As in $\S 2$ we identify $x$ with the Möbius surface $\lambda=L \circ(x, n): M \rightarrow \Lambda$. From (3.19), (4.6) and (4.7) we have

Proposition 5.1. $x$ is a Dupin surface if and only if its Möbius invariant system $\left(E_{1}, E_{2}, \Phi\right)$ satisfies $\left[E_{1}, E_{2}\right]=0$ and $\Phi=$ constant.

Thus for any Dupin surface $x$ we can find a global coordinates $(u, v)$ for $M$ defined on a domain of $\mathbb{R}^{2}$ such that $E_{1}=\partial / \partial u, E_{2}=\partial / \partial v$. So $\lambda: M \rightarrow \Lambda$ is a part of the Möbius surface $\lambda: \mathbb{R}^{2} \rightarrow \Lambda$ with the Möbius invariant system $(\partial / \partial u, \partial / \partial v, \Phi)$ with $\Phi=$ constant.

Proposition 5.2. $\lambda(\partial / \partial u, \partial / \partial v, \Phi)=\lambda\left(\partial / \partial u, \partial / \partial v, \Phi^{\prime}\right)$ in $\mathfrak{M} \ddot{o}\left(\mathbb{R}^{2}\right)$ if and only if $\Phi=1-\Phi^{\prime}$.

The proof is left to the reader. Thus there is a 1-1 correspondence between the Möbius equivalent classes of Dupin surfaces and the real numbers $1 / 2 \leq \Phi<+\infty$. Now we come to determine $\lambda(\Phi)=\lambda(\partial / \partial u, \partial / \partial v, \Phi)$. Since $S=T=0$ and $\Phi=$ constant, by (4.4) we have

$$
\frac{\partial a}{\partial u}=\frac{\partial b}{\partial v}=0
$$




$$
\begin{aligned}
\frac{\partial^{2} a}{\partial v^{2}} & =c-\frac{1}{2} \Phi a, & \frac{\partial^{2} b}{\partial u^{2}} & =d-\frac{1}{2}(1-\Phi) b \\
\frac{\partial c}{\partial v} & =-\frac{1}{2} \Phi \frac{\partial a}{\partial v}, & \frac{\partial d}{\partial u} & =-\frac{1}{2}(1-\Phi) \frac{\partial b}{\partial u} .
\end{aligned}
$$

Thus $a$ is a function of $v$ and $b$ is a function of $u$ satisfying

$$
\frac{\partial^{3} a}{\partial v^{3}}+\Phi \frac{\partial a}{\partial v}=0, \quad \frac{\partial^{3} b}{\partial u^{3}}+(1-\Phi) \frac{\partial b}{\partial u}=0
$$

Furthermore, our solution $(a, b)$ should satisfy

$$
\langle a, a\rangle=\langle a, b\rangle=\langle b, b\rangle=0, \quad\langle d a, b\rangle=0 .
$$

From these equations we can easily obtain an adapted solution $(a, b)$ in the following three cases:

Case I. $\frac{1}{2} \leq \Phi<1$.

$$
\begin{aligned}
& a=\left(0,0, \frac{1}{\sqrt{\Phi}} \cos (\sqrt{\Phi} v), \frac{1}{\sqrt{\Phi}} \sin (\sqrt{\Phi} v), \sqrt{\frac{1-\Phi}{\Phi}}, 1\right) \\
& b=\left(-\frac{1}{\sqrt{1-\Phi}} \cos (\sqrt{1-\Phi} u),-\frac{1}{\sqrt{1-\Phi}} \sin (\sqrt{1-\Phi} u), 0,0,-\sqrt{\frac{\Phi}{1-\Phi}}, 1\right) .
\end{aligned}
$$

Then by (2.8) we obtain the Dupin surface $x: \mathbb{R}^{2} \rightarrow S^{3}$,

$$
\begin{aligned}
& x=(\sqrt{\Phi} \cos (\sqrt{1-\Phi} u), \sqrt{\Phi} \sin (\sqrt{1-\Phi} u) \\
&\sqrt{1-\Phi} \cos (\sqrt{\Phi} v), \sqrt{1-\Phi} \sin (\sqrt{\Phi} v))
\end{aligned}
$$

The image $x\left(\mathbb{R}^{2}\right)$ is the isoparametric torus in $S^{3}$. The Clifford torus is the torus with $\Phi=1 / 2$. By the stereographic projection from $(0,0,0,1)$ $\in S^{3}$ we get the revolution torus in $E^{3}$ (see Figure 1):

$$
\begin{aligned}
x=\frac{1}{1+\sqrt{1-\Phi} \sin (\sqrt{\Phi} v)}(\sqrt{\Phi} \cos (\sqrt{1-\Phi} u), \\
\sqrt{\Phi} \sin (\sqrt{1-\Phi} u), \sqrt{1-\Phi} \cos (\sqrt{\Phi} v)) .
\end{aligned}
$$

Case II. $\Phi=1 . \quad a=(\cos v, \sin v, 0,-1,1,1)$,

$$
b=\left(0,0,-u, \frac{1}{2}\left(u^{2}-2\right),-\frac{1}{2} u^{2}, 1\right) \text {. }
$$

We get the Dupin surface $x: \mathbb{R}^{2} \rightarrow S^{3}$,

$$
x=\frac{1}{u^{2}+2}\left(2 \cos v, 2 \sin v, 2 u,-u^{2}\right) .
$$


The image of $x\left(\mathbb{R}^{2}\right)$ under the stereographic projection from $(0,0,0,1)$ of $S^{3}$ is the circular cylinder in $E^{3}$ (see Figure 2)

$$
x=(\cos v, \sin v, u) \text {. }
$$

Case III. $1<\Phi<+\infty$.

$$
\begin{aligned}
& a=\left(\frac{1}{\sqrt{\Phi}} \cos (\sqrt{\Phi} \bullet), \frac{1}{\sqrt{\Phi}} \sin (\sqrt{\Phi} \iota), \sqrt{\frac{\Phi-1}{\Phi}}, 0,0,1\right) \\
& b=\left(0,0, \sqrt{\frac{\Phi}{\Phi-1}}, \frac{1}{\sqrt{\Phi-1}} \sinh (\sqrt{\Phi-1} u),-\frac{1}{\sqrt{\Phi-1}} \cosh (\sqrt{\Phi-1} u), 1\right) .
\end{aligned}
$$

We obtain the Dupin surface $x: \mathbb{R}^{2} \rightarrow S^{3}$,

$$
\begin{aligned}
& x=\frac{1}{\cosh (\sqrt{\Phi-1} u)}\left(\sqrt{\frac{\Phi-1}{\Phi}} \cos (\sqrt{\Phi} v),\right. \\
& \left.\sqrt{\frac{\Phi-1}{\Phi}} \sin (\sqrt{\Phi v}), \frac{1}{\sqrt{\Phi}} \sinh (\sqrt{\Phi-1} u)\right) \text {. }
\end{aligned}
$$

The image of $x\left(\mathbb{R}^{2}\right)$ under the stereographic projection from $(0,0,0,1)$ of $S^{3}$ is the circular cone in $E^{3}$ (see Figure 3)

$$
\text { 1) } x=\exp (-\sqrt{\Phi-1} u)\left(\sqrt{\frac{\Phi-1}{\Phi}} \cos (\sqrt{\Phi} v), \sqrt{\frac{\Phi-1}{\Phi}} \sin (\sqrt{\Phi} v), \frac{1}{\sqrt{\Phi}}\right) \text {. }
$$

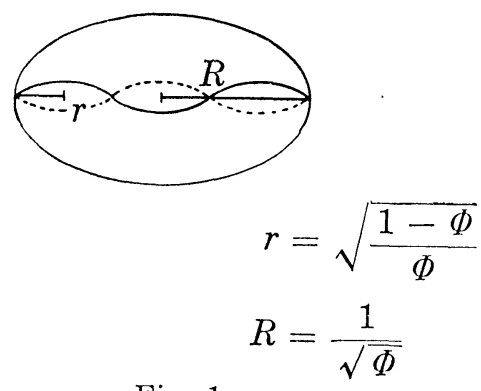

Fig. 1.

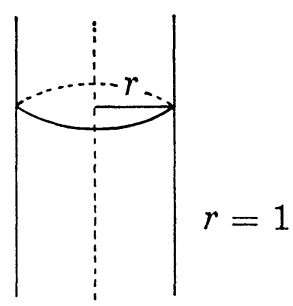

Fig. 2.

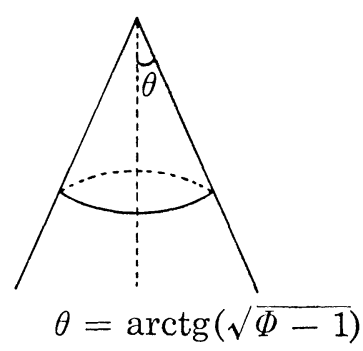

Fig. 3.

We note that the surfaces in Figures 1,2 and 3 are complete with respect to its Möbius metric.

Thus we have the following classification theorem:

TheOREM 5.3. Let $M$ be a Dupin surface in $E^{3}$. Then up to conformal transformations in $E^{3}$ it is a part of a revolution tcrus, a circular cylinder or a circular cone. 


\section{REFERENCES}

[1] R. Bryant, A duality theorem for Willmore surfaces, J. Differential Geom., 20 (1984), 23-53.

[2] T. Cecil and S. S. Chern, Tautness and Lie sphere geometry, Math. Ann., 278 (1987), 381-399.

[ 3 ] T. Cecil and S. S. Chern, Dupin submanifolds in Lie sphere geometry, Lecture Notes in Mathematics, 1369, Springer-Verlag, 1-48.

[4] T. Cecil and P. Ryan, Tight and Taut immersions of manifolds, Res. Notes Math., 107, Pitman, London, 1985.

[5] L. Eisenhart, A treatise on the differential geometry of curves and surfaces, Ginn Boston, 1909.

[6] R. Miyaoka, Compact Dupin hypersurfaces with three principal curvatures, Math. Z., 187 (1984), 433-452.

[7] B. Palmer, The conformal Gauss map and the stability of Willmore surfaces, Preprint TU Berlin, No. 267/1990.

[ 8 ] U. Pinkall, Dupin'sche Hyperflachen, Manuscr. Math., 51 (1985), 89-119.

[ 9 ] U. Pinkall, Dupin hypersurfaces, Math. Ann., 270 (1985), 427-440.

Nankai Institute of Mathematics

Nankai University

Tianjin, 300071, P.R. China

and

Technische Universität Berlin

Fachbereich Mathematik, MA 8-3

Straße des 1\%, Juni 136

1000 Berlin 12 